\title{
AN AGILE SIMHEURISTIC FOR THE STOCHASTIC TEAM TASK ASSIGNMENT AND ORIENTEERING PROBLEM: APPLICATIONS TO UNMANNED AERIAL VEHICLES
}

\author{
Javier Panadero
Angel A. Juan
Alfons Freixes \\ Javier Panadero
Angel A. Juan
Alfons Freixes \\ Javier Panadero
Angel A. Juan
Alfons Freixes \\ Universitat Oberta de Catalunya - IN3 \\ Euncet Business School \\ Carl Friedrich Gauss Av. \\ Castelldefels, 08860, SPAIN
}

\author{
Manel Grifoll \\ Carles Serrat \\ Universitat Politècnica de Catalunya \\ Dept. of Civil and Environmental Eng. \\ Dept. of Mathematics - EPSEB \\ Barcelona, 08028, SPAIN
}

Mohammad Dehghanimohamamdabadi

\author{
Northeastern University \\ Dept. of Mechanical and Industrial Engineering \\ 360 Huntington Avenue \\ Boston, MA 02115, USA
}

\begin{abstract}
Efficient coordination of unmanned aerial vehicles (UAVs) requires the solving of challenging operational problems. One of them is the integrated team task assignment and orienteering problem (TAOP). The TAOP can be seen as an extension of the well-known team orienteering problem (TOP). In the classical TOP, a homogeneous fleet of UAVs has to select and visit a subset of customers in order to maximize, subject to a maximum travel time per route, the total reward obtained from these visits. In the TAOP, a number of different tasks (customer services) have to be assigned to a fleet of heterogeneous UAVs, while the best routing plan must also be determined to cover these services. Since factors such as weather conditions might influence travel times, these are modeled as random variables. Reliability issues are also considered, since random times might prevent a route from being successfully completed before a UAV runs out of battery.
\end{abstract}

\section{INTRODUCTION}

Due to remote sensing and mobility capabilities, unmanned aerial vehicles (UAVs) are instrumental to perform a variety of civilian missions nowadays. With the recent advancement in IoT technologies, UAVs can be used for remote sensing and monitoring applications. Equipped with proper accessories and sensors, UAVs can collect and transmit data in real time. These advantages make UAVs a favorable platform for monitoring and emergency response operations. One of the promising applications of UAVs is in the maritime surveillance domain. Wide-area monitoring capacity, along with ground-based maneuvering capabilities, give UAVs a distinct advantage over manned aircraft in maritime surveillance operations. These operations can range over data acquisition (Eisenbeiss 2004), vessel classification and detection (Stacy et al. 2002), coastal surveillance to protect national borders from illegal immigrants and illicit drugs (Stone and Clarke 2001), or even locating and tracking ocean debris (Rubio et al. 2004). 
There is an increasing demand in using UAVs for surveillance and information-gathering tasks. Several examples can be found in the fields of environmental monitoring (Schaub et al. 2018), natural disaster scenarios such as floods or forest fires (Alexis et al. 2009; Popescu et al. 2015), or marine surveillance scenarios (Bürkle and Essendorfer 2010). These tasks cover exploration and data acquisition, and have the common point of avoiding the cost of direct human control.

Albeit these appealing advantages, planning and coordinating a set of UAVs to efficiently perform any of these operations require a considerable dexterity. In practice, UAVs have a limited battery capacity. Therefore, it is not feasible to keep them in service for a long time and cover the entire surveillance region. This highlights the importance of the team orienteering problem (TOP) and its applications in the context of UAVs (Bayliss et al. 2020). In this problem, the desirable goal is to dispatch UAVs to areas or service nodes that are reachable with a significant service reward value. While maximizing the total reward collected by the UAVs, subject to a maximum time allowed per route, many technical factors need to be considered, e.g., travel times between nodes, rewards associated with visiting each node, capacity of UAV batteries, etc. In addition, some unforeseen factors such as harsh weather conditions could interfere with theb UAVs' performance and the quality of the transmitted data. For instance, taking a picture of good quality might take longer than usual under windy or rainy conditions (Panadero et al. 2020), or the travel times might be affected by the wind speed. Moreover, in case of emergency needs, it is essential to plan the UAVs' routes in a timely manner with high accuracy. That emphasizes the agility requirement of the solution approach to respond quickly and solve the problem in a reliable way.

One of the stochastic factors in this problem is the traveling time which can get impacted by the service time variability and some external factors such as weather conditions. Wind variables provide information that might be used by UAV applications on marine search and rescue missions. For instance, the central region of the Mediterranean Sea is a hot spot regarding rescue operations. These operations may benefit from optimized UAV operations that maximize the total 'reward' collected with limited resources. The routes design may be a function of the wind drag. Hence, wind predictions provided by the Copernicus service are of noticeable interest. Figure 1 shows the wind predictions on a central region of the Mediterranean Sea provided by the product WIND-GLO-WIND-L4-NRT-OBSERVATIONS-012-004 on January 19 2020. The estimation of the 6-hour blended wind products make use of remotely sensed surface wind derived from real-time data provided by scatterometers located on satellites.

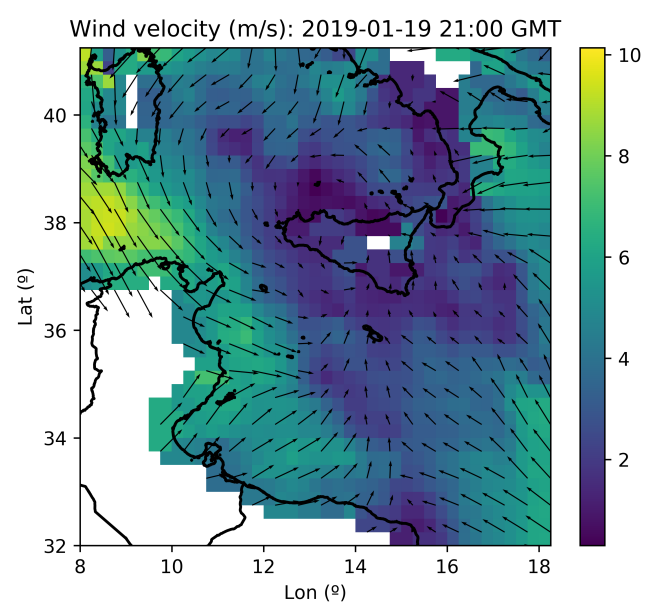

Figure 1: Wind predictions on a central region of the Mediterranean Sea.

Hence, an accurate solution approach has to properly address service time stochasticity and include the wind predictions into the calculation. Another crucial aspect of the problem is the UAVs' driving-range limitations. It is essential to provide a routing solution that ensures a high probability of task completion 
before the UAVs' battery outage. When a UAV performs the tasks that are assigned to it, its battery level gets reduced. After visiting the last node in its route, the UAV's battery level should be sufficient to enable its return to the depot.

This paper aims to solve an extension of the TOP problem considering a fleet of UAVs with heterogeneous tasks. This indicates, disregarding technical differences of the UAVs, that they are all required to perform the same tasks and provide similar services. The user's demands are heterogeneous and may need to be satisfied by multiple UAV visits. Therefore, this problem is considered as a task assignment and orienteering problem (TAOP) with uncertainty conditions. This TAOP is an integration of two different optimization sub-problems: (i) It assigns a number of different customer services among a heterogeneous fleet of UAVs and(ii) it determines the most efficient route and sequence to visit users and complete their service demand. A visual representation of this TAOP is shown in Figure 2.

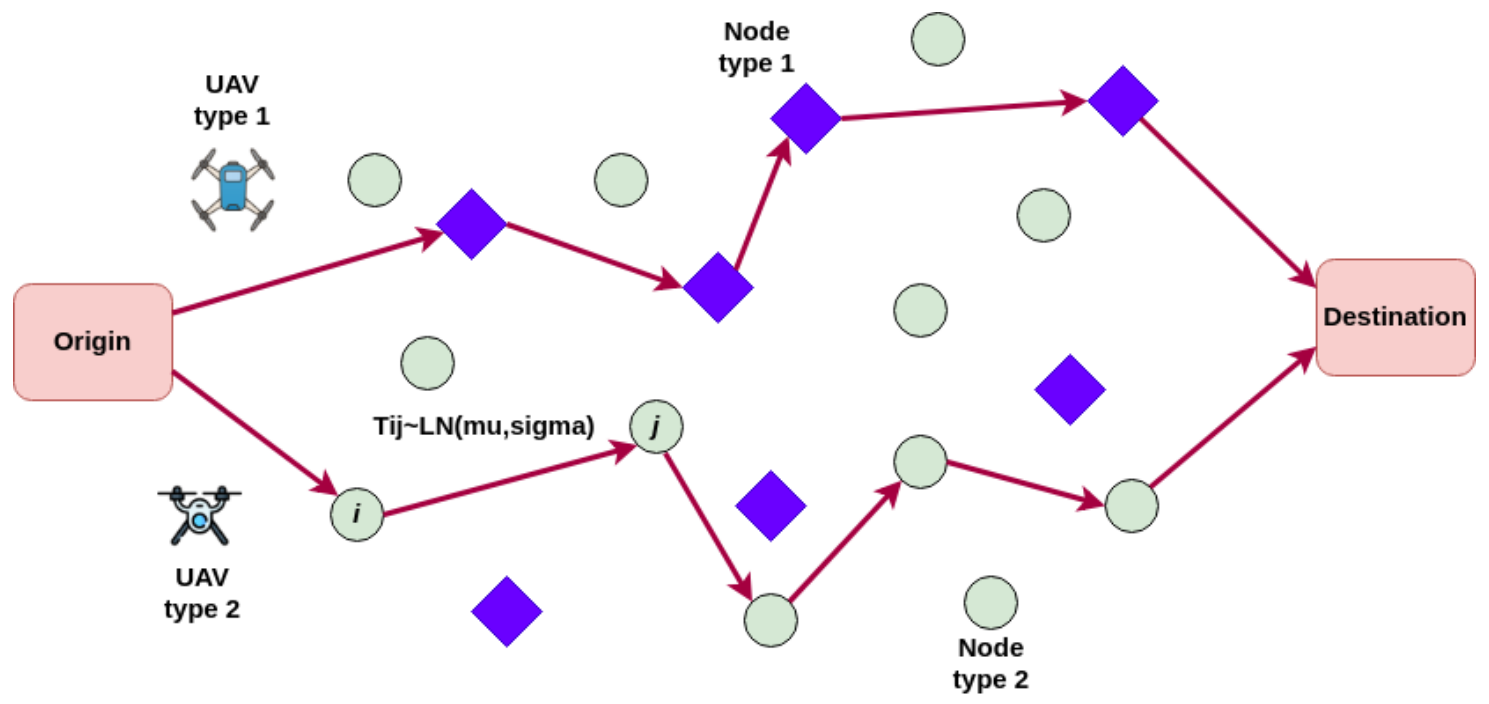

Figure 2: A visual representation of the TAOP of UAVs (without mixed-type nodes).

The main contribution of this paper is the proposal of an 'agile' (fast and flexible) simheuristic algorithm that can take into account all the aforementioned deterministic and stochastic factors. Simheuristics are a fruitful combination of heuristic algorithms with simulation models, and have been successfully employed to solve stochastic optimization problems in different areas, such as transportation (Reyes-Rubiano et al. 2019), aircraft turnaround operations (Tomasella et al. 2019), waste collection management (Gruler et al. 2017), disaster management (Yazdani et al. 2020), healthcare operations (Dehghanimohammadabadi and Kabadayi 2020), or computational finance (Panadero et al. 2018).

The remaining sections of the paper are structured as follows: Section 2 briefly reviews related articles. Section 3 describes the proposed simheuristic algorithm and its structure. Section 4 carries out a series of computational experiments to illustrate the performance of the proposed algorithm. Finally, the main findings and future research lines are given in Section 5.

\section{RELATED WORK ON UAV TASK-ASSIGNMENT AND ROUTING PROBLEMS}

Task allocation among groups of UAVs is a challenge that has been investigated during the last two decades (Jin et al. 2004). Many researchers have also analyzed the team orienteering problem (Vansteenwegen et al. 2011). Exact optimization methods, such as branch-and-bound, branch-and-cut, and dynamic programming have been used to solve small-sized instances of these problems to optimality (Keshtkaran et al. 2016). However, being NP-hard optimization problems (even in their deterministic versions), several approximate methods have been proposed to deal with large-sized instances of these two problems. Metaheuristic 
algorithms, for instance, have been used to provide near-optimal solutions to large-sized TOP instances (Archetti et al. 2007; Vidal et al. 2013; Ke et al. 2016), although they also require higher computational times than simpler heuristics. Gunawan et al. (2016) provide an excellent review of the orienteering problem and its variants.

Regarding task allocation in UAVs, iterative network flow algorithms - in which tasks are assigned to UAVs sequentially in a greedy fashion - have also been in the focus of research (Hu et al. 2015). Again, heuristic methods can perform the assignment very rapidly compared to other existing approaches. However, they can generate plans that are far from optimal. Approaches to the assignment problem that emphasize timing constraints have also been proposed (Schumacher et al. 2004). In these approaches, detailed paths are selected for each of the vehicles in order to guarantee simultaneous arrival at an anti-aircraft defense system, while minimizing exposure to radar along the way. However, these methods require that task assignment and trajectory design are solved simultaneously, which increases the problem size.

Several papers discuss task assignment under uncertainty scenarios. In Alighanbari and How (2008), the authors presented a new formulation for the UAV task-assignment problem for uncertain and dynamic environments. They proposed an alternative strategy that combines robust planning with the techniques developed to eliminate churning. The resulting robust filter embedded task assignment uses both proactive and reactive techniques to handle the uncertainty in the information, and is shown to improve worst-case behavior of the plans while, at the same time, ensuring that limited churning behavior is exhibited by the vehicle responding to noisy measurements. Choi et al. (2009) addressed single and multiple assignment problems by presenting two decentralized algorithms. Bertuccelli et al. (2009) extended one of these algorithms to solve the heterogeneous UAVs' real-time task-assignment problem under uncertainty. When executing multiple missions, UAVs form teams and are able to work cooperatively. In this context, the multi-UAV cooperative control and decision mechanisms, including task assignment, path planning, and tactical decision making, have received a great deal of attention (Chen et al. 2018). Methods like linear programming, dynamic programming, and Markov decision processes have been employed in the multi-UAV task-assignment literature (Chen et al. 2014). While centralized task assignment for multi-UAV is often not practical due to communication limits, robustness issues, and scalability, the decentralized multi-UAV task assignment problem has been also studied by Kwak et al. (2013). These authors investigated the optimization of the decentralized task assignment for heterogeneous UAVs. In their work, each UAV selects its targets by employing the consensus-based bundle algorithm. They used a scoring matrix to reflect heterogeneity among the UAVs and targets with different capabilities. In Edison and Shima (2011), a cooperative multiple task assignment problem was built up for heterogeneous UAVs performing classification, attack, and verification tasks. Zhu et al. (2018) focused on the reconnaissance task-allocation problem for UAVs, where ground targets with different features and sizes where considered. Recent publications related to stochastic TOPs are those provided by Panadero et al. (2020) and Bayliss et al. (2020). The former introduces random processing times into the analysis of TOPs, while the latter proposes a learnheuristic algorithm that considers the UAVs' physical constraints. However, none of the above analyze the integrated task-assignment and routing problem discussed in this work.

\section{AN 'AGILE' SIMHEURISTIC APPROACH}

Agile optimization techniques are crucial for real-time decision-making problems. Their algorithm design has to be fast in execution, simple in implementation, easy to tune, and flexible. In order to solve the team task-assignment orienteering problem with stochastic travel times, we propose the use of an 'agile' two-stage simheuristic algorithm, capable of providing good-quality solutions in a reasonable amount of time, even for large-scale TAOP instances with random travel times. Simheuristics are a special type of simulation-optimization algorithms that combine simulation techniques with metaheuristics (Juan et al. 2018). Depending on the characteristics of the system under considerations, simheuristics include a Monte Carlo simulation (Gonzalez-Neira et al. 2017) or a discrete-event simulation (Rabe et al. 2020). The algorithm employed in this case to solve the stochastic TAOP is described next and illustrated in Figure 3. 
During the first stage (first two rows in the aforementioned figure), the following loop is executed until a stopping criterion is met:

- A round-robin selection process is used to randomly assign a set of compatible nodes to each UAV. A biased-randomized heuristic is employed during the round-robin process. This heuristic determines the sequence of customers that the UAV has to visit. This prioritization process is based on two factors: (i) customers' compatibility with the UAV capabilities and (ii) marginal distance between each UAV's customers. The first factor ensures that the UAV can provide the expected services based on the customers' needs. The second criterion refers to the difference (in absolute

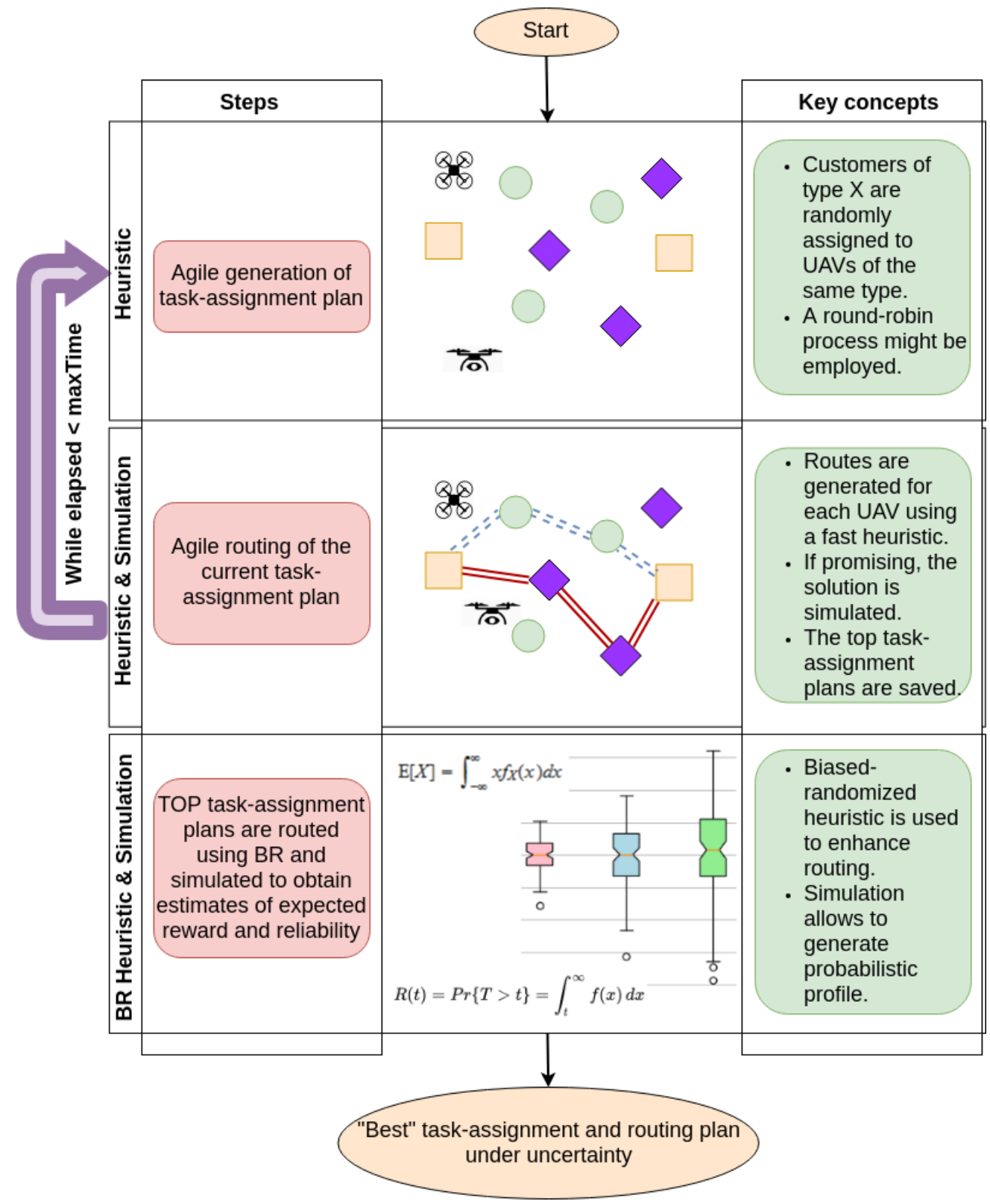

Figure 3: Schema of our simheuristic approach. 


\section{Panadero, Juan, Grifoll, Dehghanimohamamdabadi, Freixes, and Serrat}

value) between k-to-customer distance and $\mathrm{k}^{*}$-to-customer distance, where $k$ is the UAV index and $k^{*}$ represents the closest UAV to the customer without considering $\mathrm{k}$.

- Once a random but feasible task-assignment map has been constructed, the global deterministic TOP is split into smaller deterministic sub-problems (one per type of UAV), and a fast routing heuristic is employed to find the maximum reward routing plan, i.e., the best routing plan for each UAV.

- If the global team assignment and routing plan (the one resulting from the aggregation of the plans obtained for each sub-problem) provides a 'promising' reward, then a fast Monte Carlo simulation (with a reduced number of runs) is employed to test the quality of this plan when applied in a stochastic scenario (notice that rough estimates of expected total reward, reliability level, and other statistics are provided at this stage by the simulation component).

- Finally, a reduced number of task-assignment plans offering the best rough estimates are saved in a list of 'elite' task-assignment plans.

During the second stage (third row in Figure 3), these elite task-assignment plans are intensively analyzed to improve them as much as possible, thus obtaining more accurate estimates, i.e.:

- A biased-randomized TOP algorithm (Quintero-Araujo et al. 2017) is applied to each of the taskassignment plans in order to enhance the quality of the routing process and increase the associated rewards as much as possible. To maximize the total collected reward, due to a driving-range constraint, not all the nodes can be visited. Consequently, a subset of available nodes have to be selected. Biased-randomization techniques make use of a skewed probability distribution to introduce some 'smooth' randomness into the logic of the heuristic procedure, thus transforming it into a probabilistic algorithm. Applications of these techniques can be found in different optimization problems, from health care logistics (Fikar et al. 2016) to arc routing problems (Gonzalez-Martin et al. 2012).

- An intensive Monte Carlo simulation (including additional runs) is employed to generate a probabilistic profile of the associated solution, including expected total reward, reliability level, variance, quartiles, etc.

\section{COMPUTATIONAL RESULTS}

Our simheuristic algorithm was implemented as a Java application using the Eclipse IDE (Figure 4). The experiments were performed on a personal computer with $8 \mathrm{~GB}$ of RAM and an Intel Core $i 7$ at $2.3 \mathrm{GHz}$. Since, to the best of our knowledge, there are no publicly available instances for the stochastic TAOP, we decided to extend the standard instances initially introduced for the deterministic version of the TOP by Chao et al. (1996). These instances have been widely used in previous research to test the performance of algorithms aimed at solving the deterministic TOP. The extension incorporates both task assignment to each UAV, as well as random traveling times.

Regarding the task assignment, we have considered that there are two different types of UAVs (i.e., UAVs with different technical specifications), each of them performing a specific task. Hence, each type of UAV can visit a subset of customers, depending on the required task. In addition, we have randomly assigned a task to be performed to each customer. Also, for each pair of nodes $i$ and $j$, we have assumed that the travel time between them, $T_{i j}$, follows a log-normal probability distribution - in a real-world application, historical data could be used to determine the best-fit probability distribution for each of the $T_{i j}$. The log-normal distribution $T_{i j}$ has two parameters, namely the location parameter, $\mu_{i j}$, and the scale parameter, $\sigma_{i j}$, which relate to the expected value $E\left[T_{i j}\right]$ and the variance $\operatorname{Var}\left[T_{i j}\right]$ as follows: 


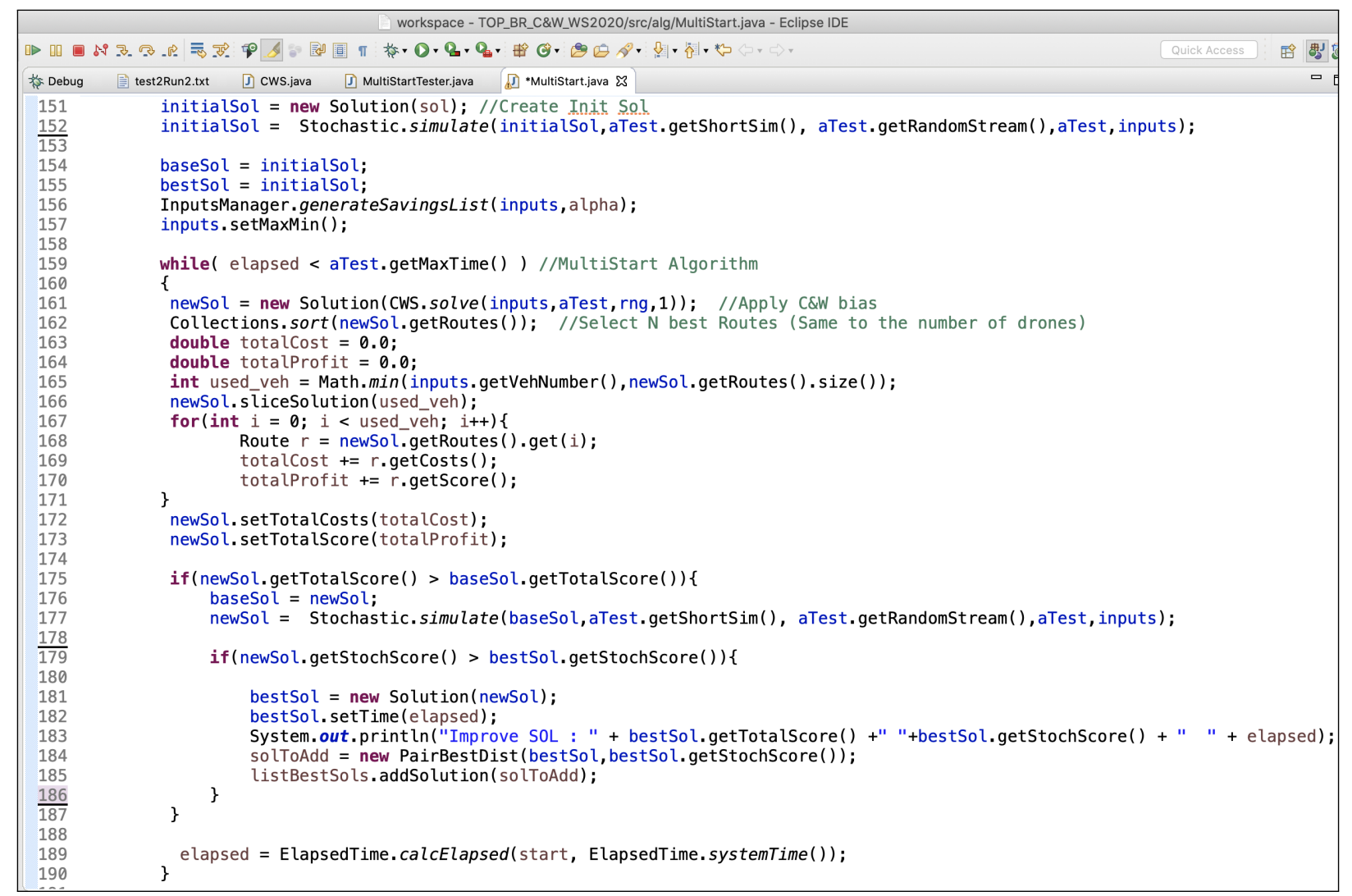

Figure 4: Screenshot of the algorithm code in the Java Eclipse IDE.

$$
\begin{gathered}
\mu_{i j}=\ln \left(E\left[T_{i j}\right]\right)-\frac{1}{2} \ln \left(1+\frac{\operatorname{Var}\left[T_{i j}\right]}{E\left[T_{i j}\right]^{2}}\right) \\
\sigma_{i j}=\left|\sqrt{\ln \left(1+\frac{\operatorname{Var}\left[T_{i j}\right]}{E\left[T_{i j}\right]^{2}}\right)}\right|
\end{gathered}
$$

In addition, we consider that $\operatorname{Var}\left[T_{i j}\right]=c \cdot E\left[T_{i j}\right]$, being $c \geq 0$ a design parameter that allows us to experiment with different levels of uncertainty. In our experiments we have considered three different levels of uncertainty: low ( $\mathrm{L}$, with $c=0.05$ ), medium ( $\mathrm{M}$, with $c=0.25)$, and large ( $\mathrm{L}$, with $c=0.50)$. The algorithm was executed five times with different seeds, storing only the best solutions in each run. A maximum time of 100 seconds was allowed for each execution. Table 1 presents the results for some selected instances with different characteristics. The first column of Table 1 identifies the instances. Each instance is characterized by following the nomenclature px.y.z.w, where: $x$ denotes the set - each set depicts a concrete scenario with a specific number of nodes and their locations, $y$ is the number of UAVs (which varies between 2 and 4 depending to the instance), $z$ indicates the number of drones of each type, and $w$ represents the maximum driving range. The second column shows the maximum travel time allowed, $T_{\max }$, while column three indicates the best-found solution (in terms of collected rewards) to the deterministic version of the problem $(O B D)$. We have divided the remaining columns into three different parts. In the first part, we evaluate our best deterministic solution under a stochastic scenario using different levels of uncertainty. Columns $O B D-x$, with $x \in\{L, M, H\}$, show the expected rewards collected when $O B D$ is evaluated in a stochastic scenario. To compute these columns, we have executed the algorithm using the expected reward of each node as a deterministic value, and disabling the simulation component. Once the 


\section{Panadero, Juan, Grifoll, Dehghanimohamamdabadi, Freixes, and Serrat}

solution has been obtained, we have estimated its real value in a stochastic environment by using simulation. In the second part of Table 1, we show the expected rewards obtained using the solution provided by our simheuristic approach, $O B S$ - $x$. Finally, in the third part, the last columns report the obtained gaps with respect to the $O B D$.

Table 1: Results under deterministic and stochastic scenarios for different levels of variance $(c)$.

\begin{tabular}{|lcc|ccc|ccc|}
\cline { 4 - 9 } \multicolumn{1}{l|}{} & & \multicolumn{2}{c|}{ Deterministic in Stochastic Scenario } & \multicolumn{3}{c|}{ Stochastic Scenario } \\
\hline Instance & $T_{\max }$ & OBD & OBD-L & OBD-M & OBD-H & OBS-L & OBS-M & OBS-H \\
\hline p1.2.1.q & 40 & 190 & 136.33 & 120.94 & 111.63 & 155.65 & 138.91 & 134.49 \\
p1.4.2.j & 12.5 & 60 & 47.72 & 41.61 & 38.98 & 51.07 & 45.21 & 42.25 \\
p1.4.2.k & 13.8 & 80 & 72.66 & 58.92 & 55.63 & 73.41 & 64.41 & 60.43 \\
p2.4.2.i & 9.5 & 120 & 119.04 & 113.11 & 109.85 & 119.20 & 112.78 & 108.29 \\
p3.2.1.n & 40 & 530 & 341.73 & 318.28 & 310.38 & 507.90 & 401.67 & 381.23 \\
p3.4.2.h & 12.5 & 220 & 182.12 & 154.46 & 145.51 & 183.70 & 151.19 & 144.63 \\
p4.4.2.k & 37.5 & 654 & 449.60 & 403.53 & 397.94 & 535.77 & 458.29 & 449.59 \\
p5.4.2.m & 16.2 & 550 & 297.56 & 282.86 & 276.93 & 320.42 & 283.55 & 271.83 \\
p4.4.2.m & 42.5 & 773 & 545.64 & 453.22 & 438.84 & 629.38 & 537.48 & 462.40 \\
p4.4.2.r & 55 & 1004 & 648.55 & 589.32 & 570.34 & 863.48 & 667.30 & 622.91 \\
p4.4.2.s & 35 & 994 & 688.89 & 597.12 & 586.19 & 893.77 & 707.68 & 664.01 \\
p5.2.1.w & 28.8 & 1320 & 834.48 & 803.62 & 765.60 & 1221.52 & 1021.65 & 993.93 \\
\hline & Averages: & 541.25 & 363.69 & 328.08 & 317.32 & 462.94 & 382.51 & 361.33
\end{tabular}

Figure 5 shows the box-plots of the aforementioned gaps. It is important to remark that these gaps are always negative, meaning that the $O B D$ can be seen as a lower bound in a scenario with perfect information (i.e., without uncertainty). The results show that the solutions provided by our simheuristic approach $(O B S-x)$ clearly outperform the deterministic solutions when these are simulated $(O B D-x)$. Actually, the latter can be seen as an upper bound for the expected cost. In other words, near-optimal solutions for the deterministic version of the problem might be sub-optimal solutions for the stochastic version. This key point reveals the importance of integrating simulation methods during the search process when dealing with

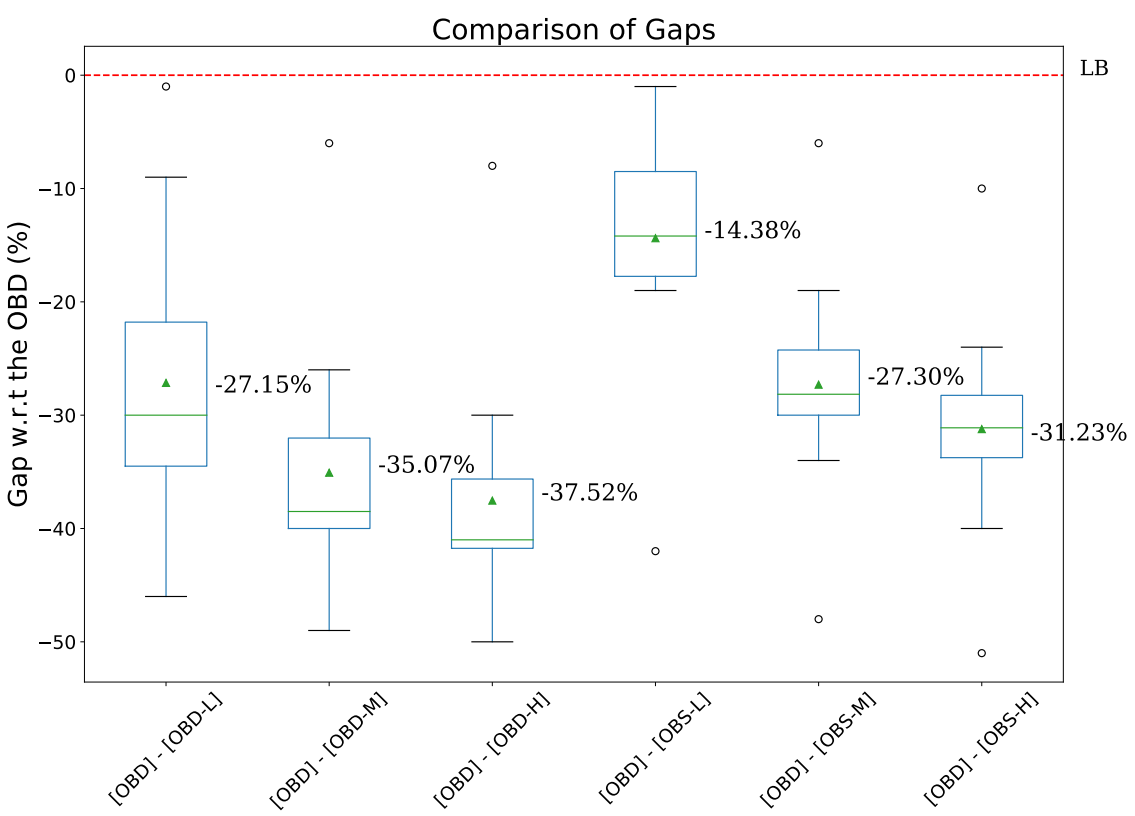

Figure 5: Boxplot of gaps w.r.t. the best deterministic solution (OBD). 
stochastic optimization problems. Regarding the reliability level of the solutions, Figure 6 demonstrates that the $O B S-x$ solutions outperform the $O B D-x$ solutions, i.e., the solutions generated by our simheuristics are more reliable. Finally, it is visible that a higher degree of uncertainty translates into a higher expected cost and, consequently, in a lower degree of reliability.

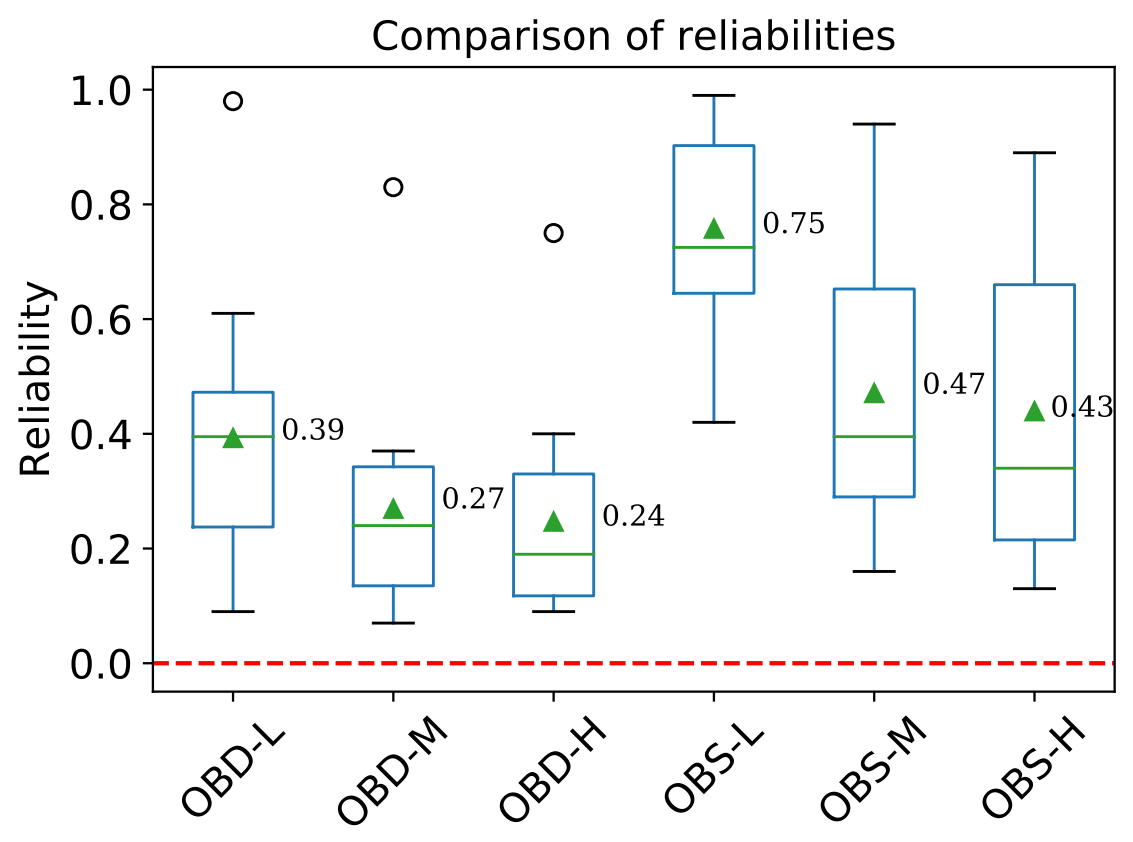

Figure 6: Boxplot of reliabilities.

\section{CONCLUSIONS AND FUTURE WORK}

This paper presents an agile simheuristic algorithm to solve a stochastic version of the team task-assignment and orienteering problem (TAOP), with a special focus on applications to unmanned aerial vehicles (UAVs). The TAOP extends the classical team orienteering problem by integrating a task-assignment stage in which a fleet of heterogeneous UAVs are assigned to customers based on their specific characteristics. Hence, the main goal is to maximize the total expected reward collected by a fleet of UAVs, while keeping an eye on the probabilistic profile of the best solutions - and, in particular, their reliability level. Both uncertainty on the travel times and the reliability of the final solutions are also taken into account.

The introduced simheuristic algorithm deals with the stochastic version of the TAOP by generating multiple task-assignment plans and then routing them using a fast biased-randomized heuristic. For each promising task-and-routing solution, associated statistics are obtained by means of a simulation process. Since travel times are considered to be random variables, the designed task-assignment and routing plans could suffer from route failures whenever the total time in covering a planned route exceeds a maximum UAV driving. Hence, not only expected times of each promising solution are computed, but also its reliability level is estimated. With this approach, the simheuristic algorithm allows the manager to select solutions with a high reward and, at the same time, a reasonably high reliability level. The performance of the proposed simheuristic is tested in an extensive experiential analysis under multiple uncertainty scenarios. The results show the superiority of the simheuristic approach over a typical metaheuristic approach. They also illustrate that near-optimal solutions for the deterministic version of an optimization problem are usually sub-optimal solutions for the stochastic counterpart. 


\section{Panadero, Juan, Grifoll, Dehghanimohamamdabadi, Freixes, and Serrat}

The approach described in this paper can be enhanced in several directions: (i) instead of using a relatively simple multi-start framework, a more advanced metaheuristic framework could be employed in order to better guide the search process if more computing time is available; and (ii) it would be interesting to analyze the effects of correlated travel times (e.g., due to common weather conditions) on the solutions provided by the simheuristics: being based on simulation, our approach could easily consider a correlation matrix between pairs of travel times.

\section{ACKNOWLEDGEMENTS}

This work has been partially supported by the Catalan Agency for Management of University and Research Grants (2017-DI-066), the Spanish Ministry of Science, Innovation, and Universities (RED2018-102642-T), the Erasmus+ program (2019-I-ES01-KA103-062602), and the Spanish Ministry of Science and Innovation (PID2019-111100RB-C21).

\section{REFERENCES}

Alexis, K., G. Nikolakopoulos, A. Tzes, and L. Dritsas. 2009. "Coordination of Helicopter UAVs for Aerial Forest-Fire Surveillance”. Applications of Intelligent Control to Engineering Systems 39:169-193.

Alighanbari, M., and J. P. How. 2008. "A Robust Approach to the UAV Task Assignment Problem". International Journal of Robust and Nonlinear Control: IFAC-Affiliated Journal 18(2):118-134.

Archetti, C., A. Hertz, and M. G. Speranza. 2007. "Metaheuristics for the Team Orienteering Problem". Journal of Heuristics 13(1):49-76.

Bayliss, C., A. A. Juan, C. S. Currie, and J. Panadero. 2020. "A Learnheuristic Approach for the Team Orienteering Problem with Aerial Drone Motion Constraints". Applied Soft Computing 92:106280.

Bertuccelli, L., H.-L. Choi, P. Cho, and J. How. 2009. "Real-Time Multi-UAV Task Assignment in Dynamic and Uncertain Environments". In AIAA Guidance, Navigation, and Control Conference, 5776. AIAA.

Bürkle, A., and B. Essendorfer. 2010. "Maritime Surveillance with Integrated Systems”. In 2010 International WaterSide Security Conference, doi:10.1109/WSSC.2010.5730231. Piscataway, New Jersey: Institute of Electrical and Electronics Engineers, Inc.

Chao, I.-M., B. L. Golden, and E. A. Wasil. 1996. "The Team Orienteering Problem". European Journal of Operational Research 88(3):464-474.

Chen, Y., D. Yang, and J. Yu. 2018. "Multi-UAV Task Assignment with Parameter and Time-Sensitive Uncertainties Using Modified Two-Part Wolf Pack Search Algorithm”. IEEE Transactions on Aerospace and Electronic Systems 54(6):2853-2872.

Chen, Y. F., N. K. Ure, G. Chowdhary, J. P. How, and J. Vian. 2014. "Planning for Large-Scale Multiagent Problems via Hierarchical Decomposition with Applications to UAV Health Management”. In 2014 American Control Conference. June $4^{\text {th }}-6^{\text {th }}$, Portland, Oregon, 1279-1285.

Choi, H.-L., L. Brunet, and J. P. How. 2009. "Consensus-based Decentralized Auctions for Robust Task Allocation". IEEE Transactions on Robotics 25(4):912-926.

Dehghanimohammadabadi, M., and N. Kabadayi. 2020. "A Combined Multi-Objective Simulation-Optimization and AHP Approach: A Healthcare Caes Study”. International Journal of the Analytic Hierarchy Process 12(1):1-20.

Edison, E., and T. Shima. 2011. "Integrated Task Assignment and Path Optimization for Cooperating Uninhabited Aerial Vehicles using Genetic Algorithms". Computers \& Operations Research 38(1):340-356.

Eisenbeiss, H. 2004. "A Mini Unmanned Aerial Vehicle (UAV): System Overview and Image Acquisition”. International Archives of Photogrammetry. Remote Sensing and Spatial Information Sciences 36(5/W1):1-7.

Fikar, C., A. A. Juan, E. Martinez, and P. Hirsch. 2016. "A Discrete-Event Driven Metaheuristic for Dynamic Home Service Routing with Synchronised Trip Sharing". European Journal of Industrial Engineering 10(3):323-340.

Gonzalez-Martin, S., A. A. Juan, D. Riera, Q. Castella, R. Muñoz, and A. Perez. 2012. "Development and Assessment of the SHARP and RandSHARP Algorithms for the Arc Routing Problem”. AI Communications 25(2):173-189.

Gonzalez-Neira, E. M., D. Ferone, S. Hatami, and A. A. Juan. 2017. "A Biased-Randomized Simheuristic for the Distributed Assembly Permutation Flowshop Problem with Stochastic Processing Times". Simulation Modelling Practice and Theory 79:23-36.

Gruler, A., C. Fikar, A. A. Juan, P. Hirsch, and C. Contreras-Bolton. 2017. "Supporting Multi-Depot and Stochastic Waste Collection Management in Clustered Urban Areas via Simulation-Optimization”. Journal of Simulation 11(1):11-19.

Gunawan, A., H. Lau, and P. Vansteenwegen. 2016. "Orienteering Problem: A Survey of Recent Variants, Solution Approaches and Applications". European Journal of Operational Research 255:315-332. 


\section{Panadero, Juan, Grifoll, Dehghanimohamamdabadi, Freixes, and Serrat}

Hu, X., H. Ma, Q. Ye, and H. Luo. 2015. "Hierarchical Method of Task Assignment for Multiple Cooperating UAV Teams". Journal of Systems Engineering and Electronics 26(5):1000-1009.

Jin, Y., M. M. Polycarpou, and A. A. Minai. 2004. "Cooperative Real-Time Task Allocation Among Groups of UAVs". In Recent Developments in Cooperative Control and Optimization, edited by S. Butenko, R. Murphey, and P. Pardalos, $207-224$. Boston, Massachusetts: Springer.

Juan, A. A., W. D. Kelton, C. S. Currie, and J. Faulin. 2018. "Simheuristics Applications: Dealing with Uncertainty in Logistics, Transportation, and Other Supply Chain Areas". In Proceedings of the 2018 Winter Simulation Conference, edited by M. Rabe, A. A. Juan, N. Mustafee, A. Skoogh, S. Jain, and B. Johansson, 3048-3059. Piscataway, New Jersey: Institute of Electrical and Electronics Engineers, Inc.

Ke, L., L. Zhai, J. Li, and F. T. Chan. 2016. "Pareto Mimic Algorithm: An Approach to the Team Orienteering Problem". Omega 61:155-166.

Keshtkaran, M., K. Ziarati, A. Bettinelli, and D. Vigo. 2016. "Enhanced Exact Solution Methods for the Team Orienteering Problem". International Journal of Production Research 54(2):591-601.

Kwak, D. J., S. Moon, S. Kim, and H. J. Kim. 2013. "Optimization of Decentralized Task Assignment for Heterogeneous UAVs". IFAC Proceedings Volumes 46(11):251-256.

Panadero, J., C. Currie, A. A. Juan, and C. Bayliss. 2020. "Maximizing Reward from a Team of Surveillance Drones under Uncertainty Conditions: A Simheuristic Approach". European Journal of Industrial Engineering 14(3):1-23.

Panadero, J., J. Doering, R. Kizys, A. A. Juan, and A. Fito. 2018. "A Variable Neighborhood Search Simheuristic for Project Portfolio Selection under Uncertainty". Journal of Heuristics 26(3):353-375.

Popescu, D., L. Ichim, and T. Caramihale. 2015. "Flood Areas Detection based on UAV Surveillance System". In 19 $9^{\text {th }}$ International Conference on System Theory, Control and Computing (ICSTCC), 753-758, doi:10.1109/ICSTCC.2015.7321384. Piscataway, New Jersey: Institute of Electrical and Electronics Engineers, Inc.

Quintero-Araujo, C. L., J. P. Caballero-Villalobos, A. A. Juan, and J. R. Montoya-Torres. 2017. "A Biased-Randomized Metaheuristic for the Capacitated Location Routing Problem". International Transactions in Operational Research 24(5):10791098.

Rabe, M., M. Deininger, and A. A. Juan. 2020. "Speeding Up Computational Times in Simheuristics Combining Genetic Algorithms with Discrete-Event Simulation". Simulation Modelling Practice and Theory 103:102089.

Reyes-Rubiano, L., D. Ferone, A. A. Juan, and J. Faulin. 2019. "A Simheuristic for Routing Electric Vehicles with Limited Driving Ranges and Stochastic Travel Times". SORT-Statistics and Operations Research Transactions 1(1):3-24.

Rubio, J. C., J. Vagners, and R. Rysdyk. 2004. "Adaptive Path Planning for Autonomous UAV Oceanic Search Missions". In AIAA 1st Intelligent Systems Technical Conference, Number 6228, 1-10. Reston, Vancouver: AIAA.

Schaub, J., B. P. Hunt, E. A. Pakhomov, K. Holmes, Y. Lu, and L. Quayle. 2018. "Using Unmanned Aerial Vehicles (UAVs) to Measure Jellyfish Aggregations". Marine Ecology Progress Series 591:29-36.

Schumacher, C., P. Chandler, M. Pachter, and L. Pachter. 2004. "UAV Task Assignment with Timing Constraints via MixedInteger Linear Programming". In AIAA $3^{\text {rd }}$ "Unmanned Unlimited" Technical Conference, Workshop and Exhibit. September $20^{\text {th }}-23^{\text {rd }}$, Chicago, Illinois, doi:10.2514/6.2004-6410.

Stacy, N., D. Craig, J. Staromlynska, and R. Smith. 2002. "The Global Hawk UAV Australian Deployment: Imaging Radar Sensor Modifications and Employment for Maritime Surveillance”. In IEEE International Geoscience and Remote Sensing Symposium, Volume 2, 699-701, doi:10.1109/IGARSS.2002.1025623. Piscataway, New Jersey: Institute of Electrical and Electronics Engineers, Inc.

Stone, R., and G. Clarke. 2001. "The T-Wing: a VTOL UAV for Defense and Civilian Applications". In UAV Australia Conference. February $8^{\text {th }}-9^{\text {th }}$, Melbourne, Australia.

Tomasella, M., A. Clare, Y. S. Gök, D. Guimarans, and C. Ozturk. 2019. "Sttar: A Simheuristics-Enabled Scheme for MultiStakeholder Coordination of Aircraft Turnaround Operations". In Proceedings of the 2019 Winter Simulation Conference, edited by N. Mustafee, K. Bae, S. Lazarova-Molnar, M. Rabe, C. Szabo, P. Haas, and Y. Son, 488-499. Piscataway, New Jersey: Institute of Electrical and Electronics Engineers, Inc.

Vansteenwegen, P., W. Souffriau, and D. Van Oudheusden. 2011. "The Orienteering Problem: A Survey". European Journal of Operational Research 209(1):1-10.

Vidal, T., T. G. Crainic, M. Gendreau, and C. Prins. 2013. "A Hybrid Genetic Algorithm with Adaptive Diversity Management for a Large Class of Vehicle Routing Problems with Time-Windows". Computers \& Operations Research 40(1):475-489.

Yazdani, M., M. Mojtahedi, and M. Loosemore. 2020. "Enhancing Evacuation Response to Extreme Weather Disasters Using Public Transportation Systems: A Novel Simheuristic Approach". Journal of Computational Design and Engineering 7(2):195-210.

Zhu, W., L. Li, L. Teng, and W. Yonglu. 2018. "Multi-UAV Reconnaissance Task Allocation for Heterogeneous Targets Using an Opposition-based Genetic Algorithm with Double-Chromosome Encoding”. Chinese Journal of Aeronautics 31(2):339-350. 


\section{Panadero, Juan, Grifoll, Dehghanimohamamdabadi, Freixes, and Serrat}

\section{AUTHOR BIOGRAPHIES}

JAVIER PANADERO is an Assistant Professor of Simulation and High Performance Computing in the Computer Science, Multimedia and Telecommunication Department at the Universitat Oberta de Catalunya (Barcelona, Spain). He is also a Lecturer at the Euncet Bussines School and a member of the ICSO@IN3 research group. He holds a Ph.D. and a M.Sc. in Computer Science. His major research areas are high performance computing, modeling and analysis of parallel applications, and simheuristics. He has co-authored more than 18 articles published in journals and conference proceedings. His website address is http://www.javierpanadero.com and his email address is jpanaderom@uoc.edu.

ANGEL A. JUAN is a Full Professor of Operations Research \& Industrial Engineering in the Computer Science Department at the Universitat Oberta de Catalunya (Barcelona, Spain). He is also the Director of the ICSO research group at the Internet Interdisciplinary Institute and Lecturer at the Euncet Business School. Dr. Juan holds a Ph.D. in Industrial Engineering and an M.Sc. in Mathematics. He completed a predoctoral internship at Harvard University and postdoctoral internships at Massachusetts Institute of Technology and Georgia Institute of Technology. His main research interests include applications of simheuristics and learnheuristics in computational logistics and transportation. He has published about 100 articles in JCR-indexed journals and over 220 papers indexed in Scopus. His website address is http://ajuanp.wordpress.com and his email address is ajuanp@uoc.edu.

MANEL GRIFOLL is an Associate Professor of the Department of Civil and Environmental Engineering of the Universitat Politecnica de Catalunya (UPC-BarcelonaTECH). He holds a Ph.D. and a M.Sc. in Civil Engineering. His main research interest include multi-port traffic analysis, weather ship routing, and applied marine science. He has published more than 40 articles in JCR-indexed journals and more than 50 documents indexed in Scopus. Currently, he is the vice-dean of International Relations and Research of the Barcelona School of Nautical Studies of the UPC-BarcelonaTECH. His e-mail address is manel.grifoll@upc.edu.

MOHAMMAD DEHGHANIMOHAMMADABADI is an Assistant Teaching Professor of Mechanical and Industrial engineering at Northeastern University (Boston, USA). He received his Ph.D. in Engineering Management from Western New England University (USA) in 2016. His research is mainly focused on developing and generalizing simulation and optimization frameworks in difference disciplines such as healthcare, supply chains, and manufacturing. His website address is https: //coe.northeastern.edu/people/dehghanimohammadabadi-mohammad/ and his e-mail address is m.dehghani@northeastern.edu.

ALFONS FREIXES is the Director of the Euncet Business School (Barcelona, Spain). He holds a M.Sc. in Management of Information Technologies from La Salle - Ramon Llull University, and a B.Sc. in Industrial Engineering from the Universitat Politècnica de Catalunya-BarcelonaTECH. He is completing a Ph.D. in the AGAUR Industrial Doctorate Program at the Universitat Oberta de Catalunya. His research area focuses on applications of statistical, computational, and IoT-related methods in smart and sustainable cities, including efficient coordination of unmanned aerial vehicles. His email address is afreixes@euncet.es.

CARLES SERRAT is an Associate Professor at the Department of Mathematics at the Universitat Politècnica de CatalunyaBarcelonaTECH, at the Barcelona School of Building Construction (Catalonia, Spain). His areas of research include, but are not limited to, methodological and applied statistics as well as methaheuristics to fields like public health, construction, civil engineering, economy, logistics, and transport. Specifically, he focuses on approaches based on survival analysis techniques, longitudinal data analysis, and missing data analysis. Professor Serrat has been granted for visiting scholarships at Harvard University and Hasselt University and visiting researcher stays at Open University of Catalonia, Trinity College Dublin, Universidad Nacional de Colombia, and Universidad de La Sabana. His website address is http://cserrat.wordpress.com/ and his email address is carles.serrat@upc.edu. 